\title{
First EMC3-Eirene simulations of the TCV snowflake divertor
}

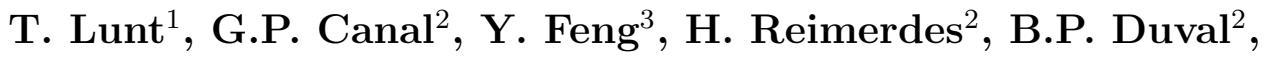 \\ B. Labit ${ }^{2}$, W.A.J. Vijvers ${ }^{2}$, D. Coster ${ }^{1}$, K. Lackner ${ }^{1}$, M. \\ Wischmeier $^{1}$ \\ ${ }^{1}$ Max-Planck-Institut für Plasmaphysik, EURATOM Association, Boltzmannstr. 2, \\ 85748 Garching, Germany \\ ${ }^{2}$ Ecole Polytechnique Fédérale de Lausanne, Centre de Recherches en Physique des \\ Plasmas, EURATOM Association, CH-1015 Lausanne, Switzerland \\ ${ }^{3}$ Max-Planck-Institut für Plasmaphysik, EURATOM Association, Wendelsteinstr. 1, \\ 17491 Greifswald, Germany \\ E-mail: tilmann.lunt@ipp.mpg.de
}

\begin{abstract}
One of the approaches to solve the heat load problem in a divertor tokamak is the so called 'snowflake' configuration, a magnetic equilibrium with two nearby x-points and two additional divertor legs. Here we report on the first EMC3Eirene simulations of plasma- and neutral particle transport in the scrape-off layer of a series of TCV snowflake equilibria with different values of $\sigma$, the distance between the x-points normalized to the minor radius of the plasma. The constant cross-field transport coefficients were chosen such that the power- and particle deposition profiles at the primary inner strike point match the Langmuir probe measurements for the $\sigma=0.1$ case. At the secondary strike point on the floor, however, a significantly larger power flux than that predicted by the simulation was measured by the probes, indicating an enhanced transport across the primary separatrix. As the ideal snowflake configuration $(\sigma=0)$ is approached, the density as well as the radiation maximum are predicted to move from the target plates upwards to the x-point by the simulation.
\end{abstract}

\section{Introduction and Motivation}

The introduction of the divertor is commonly regarded as one of the most important steps forward in fusion research. In the diverted configuration, strong density and temperature gradients can build up in the scrape-off layer (SOL), leading to more favorable conditions for the divertor target plates. In addition the SOL efficiently screens the confined plasma from impurities and facilitates the formation of an edge transport barrier. In 1982 this also lead to the discovery of the high-confinement regime (H-mode) in ASDEX [1]. However, the SOL guides a significant fraction of the total input power to the divertor target plates via parallel heat transport, which results in very localized regions of high heat flux density, $q$, around the divertor strike points (SP). These values of $q$ are already close to the acceptable limits for the divertor materials in 
present day devices, in particular during the phases of Edge Localized Modes (ELMs), an instability occurring naturally in the H-Mode [2]. Given that the volume and therefore the fusion power scales with the major radius $R$ of the machine as $R^{3}$ and the surface of the inner wall only with $R^{2}$, for purely geometrical reasons $q$ is even expected to increase significantly in large devices like ITER and DEMO. Furthermore, in present day machines it is found that the actual 'wetted area', i.e. the size of the region which receives a significant amount of power, scales only approximately linearly with $R$ (cf. Ref. [3]). Hence power exhaust remains one of the most serious problems for fusion. The 'detachment' of the power (and particle) fluxes from the divertor target plates [4] constitutes a strong relaxation of this problem. However, it is not sure, whether this state can be maintained during the entire discharge and whether the effect is sufficient in a large device.

For this reason, alternative divertor concepts are presently under investigation. At MAST the so called 'Super-X' divertor [5] is studied, where in-vessel poloidal field (PF) coils are used together with a strongly modified divertor to increase the divertor radius and to widen the field lines in front of the target, which increases the wetted area and leads to significantly longer divertor legs.

Another concept is the so called 'snowflake' divertor (SF) [6], a configuration which is characterized by a second order null-point, where not only the poloidal magnetic field but also its spatial derivatives vanish. Such a configuration is more easily achieved with PF coils close to the plasma. TCV [7, 8], NSTX [9] and DIII-D [10], which are equipped with PF coils inside the toroidal field ( $\mathrm{TF}$ ) coils, were the first to test this configuration. In a reactor, where the superconducting coils require strong shielding from the fusion neutrons, the installation of PF coils inside the TF coils is a severe technical problem. The ideal SF constitutes a particular case within a set of configurations that feature two nearby x-points. The parameter $\sigma$ is defined as the distance between the two xpoints normalized to the minor plasma radius $a$. Following the convention introduced in Refs. [6, 11], $\sigma=0$ constitutes the ideal SF. Experimentally, however, $\sigma$ will never be accurately 0 and the two x-points will never be exactly at the same poloidal flux $\Psi$. So one of the two x-points will define the 'primary' separatrix as well as the common- and the private flux region. We will then speak of a 'snowflake plus' $(\mathrm{SF}+)$ configuration if the secondary $\mathrm{x}$-point is located in the private flux region and a 'snowflake minus' (SF-) configuration if the secondary $\mathrm{x}$-point is in the common flux region.

Any SF configuration is accompanied by the appearance of two additional divertor legs and thus two additional strike points. It has been found experimentally that a significant fraction of power can be deposited at these secondary strike points for configurations with $\sigma \leq 0.6$, in particular during the ELM phases [9, 12].

While the SF configuration is presently being experimentally studied in several devices a rather small number of reports on efforts to simulate the plasma transport of this configuration numerically is found in the literature $[13,14,15]$. As a contribution to the latter this article reports on first simulations of the TCV SF divertor by means of the Edge Monte-Carlo 3D-Eirene (EMC3-Eirene) code package (cf. Sec. 3). These are also 
the first simulations whose computational domain includes the secondary separatrix. Before addressing the full transport simulations in Sec. 3 we will first study the changes of the magnetic geometry introduced by this new configuration when approaching $\sigma=0$ in Sec. 2. In Sec. 4 we will then present and discuss the results in detail and close with a summary and an outlook in Sec. 5.

\section{Features of the equilibrium during a $\sigma$-scan}

The heat flux density at the target depends on the transport properties of the plasma and on the magnetic geometry determined by the equilibrium. While the study of the edge plasma transport is a more comprehensive work addressed in Sec. 3, it is worthwhile to study the magnetic geometry first, which we will characterize here by the connection length $L_{c}$, the flux expansion $f_{x}$ and the target pitch angle $\theta_{\text {pitch }}$. The first is defined as the length of a field line crossing a point $R, z, \phi$ and two intersection points at the target surfacest, $t_{1}$ and $t_{2}$,

$$
L_{c}=\int_{t_{1}}^{t_{2}} d L_{\|}=\int_{t_{1}}^{t_{2}} \frac{B}{B_{\theta}} d L_{\theta}
$$

the second by

$$
f_{x} \equiv \frac{d r}{d r_{u}}=\frac{R_{u} B_{\theta, u}}{R B_{\theta}}
$$

where $u$ refers to the upstream position, while the third is given by

$$
\theta_{\text {pitch }} \equiv \operatorname{atan}\left(B_{\theta} / B_{\phi}\right) \approx B_{\theta} / B_{\phi}
$$

with $\phi$ and $\theta$ indicating the toroidal and poloidal directions. $d L_{\|}$is an infinitesimal length along the magnetic field line and $d L_{\theta}$ its poloidal projection. $B$ is the (total) magnetic field and $d r$ the radial distance between two neighboring flux surfaces. In the following, $L_{c}, f_{x}$ and $\theta_{\text {pitch }}$ will refer to their values at the target position, unless something else is specified explicitly. In order to reduce the power flux density at the target it is favorable to increase $L_{c}$ and $f_{x}$ and to incline the target surface with respect to the flux surfaces. Since the toroidal field $B_{\phi}$ in the whole device, as well as the poloidal field $B_{\theta}$ in the main chamber, can only be varied within a very limited range without altering the core plasma performance, $B_{\theta}$ in the divertor region itself has to be decreased. Inclining the target with respect to the flux surfaces such that the poloidal angle between the surface normal and the separatrix is $\gamma$, the radial upstream interval $d r_{u}$ is mapped to the interval

$$
d s=d r_{u} f_{x} / \cos (\gamma)
$$

on the target. Increasing $\gamma$ and thereby $d s$ reduces the power flux density there. However, the field line incident angle $\theta_{\perp}=\operatorname{atan}\left(B_{\perp} / B_{\phi}\right)=\operatorname{atan}\left(B_{\theta} \cos (\gamma) / B_{\phi}\right) \approx$

$\ddagger$ Note that some authors define $L_{c}$ as the length from the outboard (or the inboard) midplane to the divertor. Since the connections lengths in the analysed TCV equilibria calculated according to different definitions are approximately proportional, it is irrelevant which one we apply here. 
$\theta_{\text {pitch }} \cos \gamma$ may not be arbitrarily small or surface imperfections in the sub-mm range as well as inaccuracies in the tile alignment due to engineering constraints could lead to a strong toroidal inhomogeneity of the deposited power on the divertor plates. This effect is expected to play a significant role for values of $\theta_{\perp}$ smaller than $2-3^{\circ}$.

The simulations described in the following sections are based on TCV experiments in the SF+ configuration [12]. We analyze a set of equilibria with the same plasma profiles and similar plasma boundaries, but with four different values of $\sigma, 0.01,0.10,0.5$ and 1.0 (cf. Fig. 2), generated by the free boundary equilibrium code SPIDER [16]. The strike points of the divertor legs defined by the primary x-point are located on the inner and outer wall and the secondary x-point defines another two strike points, which are located on the inner wall and the floor of the TCV vessel. Neither location nor tilt angle of the divertor targets are optimized for divertor performance. The strike points are numbered counter-clockwise from $\mathrm{SP}_{1}$ to $\mathrm{SP}_{4}$ starting with the primary strike point on the inner wall. In order to characterize the magnetic geometry of the different SF equilibria, $L_{c}$, normalized poloidal flux $\Psi_{N}=\left(\Psi-\Psi_{\text {axis }}\right) /\left(\Psi_{\text {separatrix }}-\Psi_{\text {axis }}\right)$ and $\theta_{\text {pitch }}$ have been calculated as a function of the relative position of a starting point of the magnetic field line with respect to the primary strike point positions $\mathrm{SP}_{1}$ and $\mathrm{SP}_{4}$, as shown in Fig. 1.

It was argued, e.g. in [17], that the connection length of field lines in the SOL increases as $\sigma$ is reduced. For the very close vicinity of the separatrix this statement is confirmed, as shown by the insert in Fig. 1, where the interval near the SP is zoomed. However, $L_{c}$ is only increased in a tiny region of about $0.2 \mathrm{~mm}$ around the strike points. Outside this small region, $L_{c}$ is significantly shorter for configurations close to the ideal SF (solid black and dotted red curves in Fig. 1) compared to those with larger $\sigma$ (blue dash-dotted curve in Fig. 1) in TCV. (Note that the black curve corresponding to $\sigma=0.01$ is not shown in the zoom due to inaccuracies in the equilibrium/field line tracing.) However, it has been shown that the size of the region, with enhanced $L_{c}$ scales with the minor plasma radius $a$ (cf. Ref. [18]) and would be much larger in reactor size experiments like ITER and DEMO.

Additionally the flux expansion at the targets decreases, which can be seen by the fact that $\Psi_{N}$ increases slower with increasing distance to the strike point for larger $\sigma$ values. This is due to the fact that the targets in TCV are located at a significant distance to the null point, where a recompression of the flux surfaces takes place. One could take advantage from an enhanced flux expansion by placing the targets closer to the null point as it is the case in DIII-D and NSTX. This, however, would shorten the absolute value of $L_{c}$ and as a consequence it would be harder to build up a high density region (and low temperatures) in front of the target required to keep the sputtering low and the pumping efficiency high. In a reactor these criteria will have to be taken into account to find the optimum target position.

Given that an increase of $f_{x}$ is always related to a decrease of $\theta_{\text {pitch }}$ and vice versa these quantities cannot be treated independently. As shown by the bottom row of Fig. $1 \theta_{\text {pitch }}$ for $\sigma=1$ is already close to the engineering limit for $\theta_{\perp}$ so that $\gamma$ cannot be chosen very 
large. For smaller $\sigma$ values $\theta_{\text {pitch }}$ increases allowing larger target tilt angles $\gamma$. We will come back to this point later on in Sec. 4.2.

Finally, the magnetic field structure just inside the separatrix is also important for the processes in the SOL. In particular the magnetic shear in the vicinity of the plasma boundary increases in the SF configuration as shown in Fig. 6 of Ref. [7], which can have an impact on the ELMs as well as the pedestal structure. Here, however, neither time dependent phenomena nor the edge transport barrier are taken into account.

\section{EMC3-Eirene}

For the particle-, momentum- and power transport simulations the Edge Monte Carlo 3D - Eirene (EMC3-Eirene) code package was used. EMC3 solves the three dimensional (3D) Braginskii equations and is self consistently coupled to Eirene that solves the kinetic 3D Boltzmann equation for neutral particles. Detailed information on Eirene can be found in Ref. [19] while the working principle as well as the full set of equations solved by EMC3 and the coupling to Eirene are described in detail in Ref. [20]. Although a Monte Carlo principle is used to solve the equations mathematically, that EMC3 describes the edge plasma as a fluid including all the approximations of the fluid picture.

Addressing the same geometry (axisymmetric configurations) and the same physics models, benchmarks have recently shown a reasonably good agreement with the 2D fluid codes SOLPS and EDGE2D-Eirene [21, 22, 23]. Nevertheless, a limitation of the present version of EMC3-Eirene is that cross-field transport parameters $D_{\perp}$ and $\chi_{\perp}$ are assumed to be constant over the whole computational domain and that volumetric recombination, drifts and flux limiters are not (yet) included in the code.

The motivation for the application of a 3D code to a toroidally symmetric configuration, like the SF divertor, is purely technical, namely a considerably simpler construction of a computational grid for this particular case. While many 2D codes require a grid aligned to the flux surfaces, EMC3 only requires an alignment with the field lines in the toroidal direction. As an example, a grid for a $\mathrm{SF}+$ configuration with $\sigma=0.5$ is shown in Fig. 3 , where in the confinement region (red) and the SOL (blue) the grid is aligned with the magnetic flux surfaces, while the 'private flux region' (PFR, green) is covered by a single regular grid including the complicated structures around the secondary x-point (lower red cross) and the secondary separatrix (red solid line). The toroidal extension of the grid is $20^{\circ}$, i.e. $1 / 18$ of the full circumference. This value was chosen for technical reasons to run the code in an efficient way. Periodic boundary conditions are defined at the first and last toroidal plane of the computational domain.

For further technical reasons the divertor target plates (as indicated by the inner black solid line in Fig. 3) were moved several cm inwards in particular on the low field side and at the floor, as indicated by the dashed blue line in the figure. Extending the computational domain for the equilibrium would solve this issue. However, since the

shift is of the order of only $10 \%$ of the divertor leg length, the simulation with the artificial target is expected not be very different to one with the real geometry. 


\section{Simulation results and discussion}

\subsection{Experimental input parameters}

After having constructed a computational grid from the equilibrium (for the time being we focus on the $\sigma=0.1$ case, i.e. close to the ideal SF) we can now address a first simulation of the deuterium bulk plasma- and neutral particle transport by means of EMC3-Eirene. For this purpose we assume an input power of $P_{i n}=238 \mathrm{~kW}$ across the inner simulation boundary $\left(\Psi_{N}=0.9\right)$ equally distributed between the electrons and ions $P_{e}=P_{i}=0.5 P_{i n}$. A further boundary condition for the particle balance is required, which can be either the total flux to the targets or the (average) density $n_{e}$ on a particular flux surface. We chose the latter here, but instead of fixing $n_{e}$ at the separatrix as usual, we fix it at the flux surface at the inner simulation boundary and iterate the transport equations such that $n_{e}$ at the separatrix point furthest away from the $\mathrm{x}$-point is $2 \cdot 10^{19} \mathrm{~m}^{-3}$, which is approximately the value measured by Thomson Scattering in discharge \#43418 and a typical value for high recycling discharges in TCV. The reason for this choice is the fact that the density is not constant on the flux surfaces in the vicinity of the separatrix, which will be discussed later (cf. Sec. 4.3).

Transport coefficients of $D_{\perp, e}=D_{\perp, i}=\chi_{\perp, e}=\chi_{\perp, i}=0.6 \mathrm{~m}^{2} / \mathrm{s}$ were assumed in order to best match the ion saturation current $j_{\text {sat }}$ and in particular the $q$ profiles at SP 1 measured by Langmuir probes. These profiles are shown in Figs. 4 and 5, where, for the time being, we only compare the simulation for $\sigma=0.1$ that correspond to the data points measured for the same $\sigma$ value. Note that the experimental profiles at $\mathrm{SP}_{1}$ are shifted by $12 \mathrm{~mm}$ upwards, which is within the uncertainty of the equilibrium reconstruction. Furthermore, the heating power in the experiment was slightly higher $\left(P_{\text {heat }}=270 \mathrm{~kW}\right)$ while a large fraction of about $50 \%$ was measured to be lost from the bulk plasma via radiation, i.e. before reaching the target, a process not yet accounted for in the simulations. In addition to that it was found that the convergence behavior was getting worse as $\chi_{\perp}$ decreased. While the (global) change between two iterations of the temperature field is in the $1 \%$ range for $\chi_{\perp}=3 D_{\perp}=1.8 \mathrm{~m}^{2} / \mathrm{s}$, it is $\sim 3 \%$ for $\chi_{\perp}=2 D_{\perp}=1.2 \mathrm{~m}^{2} / \mathrm{s}$ and $\sim 6 \%$ for $\chi_{\perp}=D_{\perp}=0.6 \mathrm{~m}^{2} / \mathrm{s}$. The fluctuations are strongest in remote areas from the main plasma, where density and temperature are low and the Monte Carlo noise high, but are also non-negligible in the secondary divertor legs, while in the common flux region the profiles are very stable during the iteration process. In this sense the simulation is still insufficient for an accurate quantitative comparison to the experiment, however, it is rather unlikely that a more refined power balance and/or the application of a different numerical scheme to solve the transport equations will explain the enormous discrepancy between the simulated and experimentally measured particle and power fluxes to $\mathrm{SP}_{3}$, which we will discuss in the following sub-section.

Since we constructed a non-flux surface aligned computational grid in the PFR, it is particularly important to test the numerical correctness of the simulations. For this purpose we compared a simulation with a 'standard' resolution (40 radial surfaces) with another, where the radial resolution of the PFR was doubled. The particle and power 
fluxes agreed to within a few percent which is within the accuracy of the Monte Carlo noise.

\section{2. $\sigma$ scan and transport across the primary separatrix}

The same simulation as in the previous section was repeated for all equilibria analyzed in Sec. 2 and we now compare the same series of $\sigma=0.01,0.10,0.50$ and 1.00. In Sec. 2 we saw that connection length as well as flux expansion are decreasing with decreasing $\sigma$. In particular due to the latter the peak $j_{\text {sat }}$ and $q$ values increase and move towards the strike point position as $\sigma$ approaches 0 (cf. Figs. 4 and 5) in TCV, which obviously is opposite to the desired behavior. The decreasing flux expansion is almost entirely responsible for this effect, which becomes obvious from Fig. 6, where the parallel power flux $q_{\|}$is plotted versus the magnetic coordinates $\Psi-\Psi_{S P, i}$. With spatially constant transport coefficients these profiles look very similar for the different values of $\sigma$. Given that the pitch angle $\theta_{\text {pitch }}$ is increasing with decreasing $\sigma, \theta_{\perp}$ and $d s$ can be kept constant increasing the target inclination angle $\gamma$.

The comparison of the simulated power deposition profiles at $\mathrm{SP}_{3}$ to the experimental ones, shows that the power flux into the private flux region predicted by the code is at least an order of magnitude too low. The measurement therefore indicates the existence of a transport channel other than diffusion with a spatially constant coefficient, possibly driven by instabilities due to the large value of $\beta_{p}=2 \mu_{0} p / B_{\theta}^{2}$ occurring in the low $B_{\theta}$ region around the $\mathrm{x}$-point as proposed by Ryutov, who discussed these in in detail in $[24,25]$. Drifts may also play a role in the null-point region, however, here the flux surfaces are far away from each other and if the electric potential is constant on a flux surface, the radial electric field at the x-point is expected to be small. The repartition of power to the secondary SPs is accompanied by the desired reduction of the load to the primary SPs. In TCV, however, this is only a marginal effect of the order of $10 \%$. Since the size of the region with low $B_{\theta}$ scales with machine size, while $\lambda_{q}$ essentially does not, the effect might be stronger in a reactor-scale device [18]. This hypothesis, however, would need confirmation by a physical model of the instability and/or further experiments.

\subsection{Formation of a density blob}

A very interesting prediction from the simulations of the $\sigma$-scan is that the density peak, originally located in front of the target due to the strong recycling, moves upstream as $\sigma$ is reduced leading to the formation of a density blob in the null-point region. A poloidal cross section of the $n_{e}$ distribution during the $\sigma$ scan is shown in Fig. 7.

This phenomenon is explained by the hampered energy transport to the null point region for smaller $\sigma$ values. Neutrals from the large reservoir at the bottom of the machine (cf. Fig. $8(\mathrm{~d})$ ) penetrate the separatrix in the region of the x-point, where they are ionized (compare Fig. 8 (e) and Fig. 9 (e) for $\sigma=0.01$ and $\sigma=1.00$, respectively). As a consequence, the local $n_{e}$ increases and $T_{e}$ drops due to the energy loss associated with 
the ionization processes as well as the energy fluxes carried by the ionized particles when leaving this region through either parallel or cross-field transport (cf. Fig. 8 (a)). The total plasma pressure is approximately constant along flux tubes (cf. Fig. 8 (b)). For $\sigma=1$, the strong parallel heat transport is counteracting this effect suppressing even small $T_{e}$ gradients. However, due to the much smaller pitch angle in this region, for the $\sigma=0.01$ configuration, the parallel length corresponding to the same poloidal interval is increasing significantly, allowing significant poloidal $T_{e}$ gradients to build up (cf. Fig. 9 (a) which has a much smaller $T_{e}$ drop at the x-point than Fig. 8 (a)). Furthermore, the flux expansion around the x-point reduces the perpendicular diffusion fluxes for both particle and energy, supporting the density build up there further. As the cross section for ionization, $\sigma_{i}$, has little dependence on $T_{e}$ above $20 \mathrm{eV}$, the ionization rate $R_{i}=n_{e} n_{n}\left\langle\sigma_{i} v_{e}\right\rangle$, increases with increasing $n_{e}$, which amplifies the original effect. This feed back mechanism ceases when $T_{e} \lesssim 10 \mathrm{eV}$, where a further drop of $T_{e}$ results in a significant reduction of $R_{i}$.

This explanation is corroborated by several additional simulations where the influence of different effects are investigated: (A) $T_{e}$ is set to the constant value of $20 \mathrm{eV}$ in the whole computational domain, i.e. the plasma is assumed to be isothermal with no limitation in heat supply, (B) the neutrals are screened from the null point region by an additional target (this screen does not affect the plasma) to turn off the ionization source there and (C) the private flux region is screened from the neutrals. In all these simulations the density blob disappears. In a further run, (D), only the energy sink term related to the ionization and charge exchange with the neutrals was turned off in the energy equation resulting in a significantly less pronounced (about $15 \%$ ) but still existent plasma density peak, which is due to the fact that the plasma is locally cooled mainly due to the heat transported away by the ionized particles.

Another candidate to explain the formation of this density blob is the presence of flows in the SOL, which transfer momentum via viscosity to the confined plasma thus inducing flows with a poloidal component pointing towards the x-point, as can be seen in Fig. 8 (c). (Note that the particles leave the $\mathrm{x}$-point region via radial diffusion and provoke parallel flows with opposite sign further inwards in the confined region.) Although this effect apparently contributes to the particle accumulation at the null point, another simulation, (E), has shown that it is not the dominant mechanism. In (E), the neutral particles were guided artificially from the target to a SOL position upstream of the null-point where they ionize. Although these sources provoke strong convective flows in the SOL the large density peak does not form.

In these simulations we assumed constant diffusive transport coefficients. The enhanced transport at the x-point discussed in Sec. 4.2, however, may counteract the formation of this density peak, so that its occurrence in the experiment is not obvious. A measurement of the density at the x-point would therefore be a further test whether the transport in the null region is enhanced. 


\subsection{Trace impurity transport}

On TCV no experimental data for the x-point density is available so far. 2D profiles of the radiation from some impurity line like carbon on the other hand are much easier to measure by means of cameras equipped with adequate spectral filters. For this reason, we also performed trace impurity transport simulations for carbon ions with EMC3. The details of this simplified transport model, which assumes thermal equilibrium of the impurity ions with those of the bulk plasma $\left(T_{Z}=T_{i}\right)$ are given in [20]. The atomic data from the ADAS data base [26] was taken for the ionization and recombination cross sections together with those for excitation in order to deduce the carbon emissivity $\epsilon_{C}$ profile. A constant sputtering yield $Y$ was assumed, i.e. every Deuterium ion impinging on the target is sputtering $Y$ carbon atoms on average independently of the incident energy, charge state or impinging angle. Given that we are only interested in the radiation distribution here (and not its absolute value), the actual value of $Y$ is not important. The result of the four different cases of $\sigma$ is shown in Fig. 10, where the null-point and primary strike point regions are zoomed (in the rest of the plasma the emissivity is substantially smaller). One can see clearly that the carbon radiation behaves similarly to the density, i.e. the radiation peak also moves from a location in front of the target to the x-point region. Such a radiation peak was indeed experimentally observed in TCV as can be seen in Fig. 4 (a) and (b) of Ref. [7]. The SF configuration thus offers an interesting control mechanism to set the location of the maximum radiation and possibly also its amplitude. However, since the impurity radiation decreases the temperature even further it also carries the risk of the occurrence of a thermal instability.

\subsection{Power scan}

In order to test the sensitivity of the results on the external discharge parameters we scanned the input power $P_{\text {in }}$ in 4 steps, (a) $119 \mathrm{~kW}$, (b) $238 \mathrm{~kW}$, (c) $475 \mathrm{~kW}$ and (d) $713 \mathrm{~kW}$. It is found that the density peak is increasing from (a) 3.6 (b) 4.5, (c) 5.5 to (d) 6.2 times the value of the lowest density on the separatrix. Associated with the increase of input power, the position of the density maximum moves from about $5 \mathrm{~cm}$ above the null point to approximately $2 \mathrm{~cm}$ below, i.e. into the PFR, as shown in Fig. 12 (Note that the global density maximum in case (d) actually returns to the position in front of the target). The power deposition profile at the target during this scan is shown in Fig. 11. Note that the profiles are scaled with $P_{r e f} / P_{i n}$, where $P_{\text {ref }}=238$ $\mathrm{kW}$, for the sake of comparability. Since the separatrix temperature and therefore the parallel heat conductivity is slightly larger for larger input powers $P_{i n}$ in the nearSOL region the profiles are peaking stronger at higher $P_{i n}$. The numerical values of the integrated power $P_{S P_{i}}=\int_{S P_{i}} \vec{q} d \vec{A}$ to the 4 strike points $\mathrm{SP}_{1}$ to $\mathrm{SP}_{4}$ as well as the fraction transfered to neutral particles via charge exchange and elastic collisions and that leaving the computational domain radially are given in Tab. 1. Due to the stronger peaking a larger fraction of the power is going to the targets, while a smaller fraction leaves the 
computational domain at the outer simulation boundary at larger $P_{i n}$. The fraction of power that is arriving the secondary strike points $\mathrm{SP}_{2}$ and $\mathrm{SP}_{3}$ is also increasing with $P_{i n}$, this fraction, however, is still of the order of $\sim 1 \%$ even for the highest $P_{i n}=713$ $\mathrm{kW}$ in contrast to the measurement.

\section{Summary and outlook}

The enormous heat flux densities that can occur close to the strike points of a divertor tokamak are known as one of the major outstanding problems in fusion research. For this reason the snowflake divertor, a magnetic configuration with two nearby $\mathrm{x}-$ points, is presently investigated as one of the alternative concepts to solve the power exhaust problem. In this article we analyzed a series of magnetic equilibria with normalized distances $\sigma=0.01,0.10,0.50$ and 1.00 between the x-points, that are based on experiments. As a first step we analyzed the geometrical properties of these configurations, namely the connection length $L_{c}$, the flux expansion $f_{x}$ and the pitch angle $\theta_{\text {pitch }}$ at the target. It was found that the region, where $L_{c}$ in the ideal snowflake configuration exceeds the value in standard single-null configurations, corresponds only to a small region in the immediate vicinity of the separatrix corresponding to $0.2 \mathrm{~mm}$ along the target surface in TCV. Since this region is significantly smaller than the SOL width defined by the power fall-off length $\lambda_{q}$ TCV cannot take advantage of an increased $L_{c}$ in the SF configuration. In a larger machine like DEMO in contrast, where this region is much larger compared to $\lambda_{q}, L_{c}$ would be enhanced in the entire near-SOL region of high heat flux. Furthermore, the flux expansion decreases with decreasing $\sigma$, while $\theta_{\text {pitch }}$ grows. In a second step we performed a full particle, momentum, and energy transport simulation with the EMC3-Eirene code package of the deuterium plasma and the neutral gas in the TCV divertor. The simulations show a peaking of the particle- and power deposition profiles as $\sigma$ decreases, in contrast to the desired broadening. This increase is mainly explained by the decreasing flux expansion at the target plates in TCV that are rather far away from the x-point and not optimized for power exhaust. For closer target plates this effect would be less pronounced or even inverse. In any case the decrease in flux expansion can be compensated by increasing the target tilt angle. Due to the growing $\theta_{\text {pitch }}$ with decreasing $\sigma$ the targets can be inclined stronger for a snowflake configuration respecting the same engineering limits for $\theta_{\perp}$ in a reactor. This then leads to a zero net effect if the transport was purely diffusive with constant coefficients. The much larger power fluxes to the secondary $\mathrm{SP}_{3}$ found experimentally, however, indicate the existence of a radial transport channel other than diffusion with spatially constant coefficients, possibly driven by a $\beta_{p}$ instability occurring locally at the x-point. This redistribution of the power to the private flux region decreases the heat flux to the primary strike points as desired, although the effect in TCV is only in the $10 \%$ range. Due to the same argument as before a larger fraction might be re-distributed in a reactor-scale device.

Approaching the ideal snowflake configuration $(\sigma=0)$ the density maximum is found 
to move upstream in the simulation leading finally to the formation of a density blob at the null point. Since the impurity density and radiation show the same behavior, this configuration offers the possibly of controlling the radiated power in the separatrix near confinement and SOL regions, although it harbors a higher risk for the occurrence of thermal instabilities. The enhanced transport at the x-point discussed above may counteract the formation of this density peak, so that its occurrence in the experiment is not obvious. A measurement of the density at the x-point would therefore be a further test whether the transport in the null region is enhanced.

As one of the next steps a more refined power balance study including an impurity transport simulation as well as a test of an enhanced (diffusive or convective) transport localized at the $\mathrm{x}$-point is foreseen. In addition to the $\mathrm{SF}+$ configuration the $\mathrm{SF}$ configuration would be of high interest, the scaling with machine size as well as an optimized target geometry.

\section{References}

[1] Wagner F. et al. 1982 Phys. Rev. Lett. 491408

[2] Zohm H. et al. 1996 Plasma Phys. Contr. Fusion 38 105-128

[3] Eich T. et al. 2011 Phys. Rev. Lett. 107215001

[4] Shimomura Y. et al. 1983 Nucl. Fusion 23869

[5] Valanju P.M. et al. 2009 Phys. of Plasmas 16056110

[6] Ryutov D.D. et al. 2007 Phys. of Plasmas 14064502

[7] Piras F. et al. 2009 Plasma Phys. Contr. Fusion 51055009

[8] Piras F. et al. 2010 Phys. Rev. Lett. 105155003

[9] Soukhanovskii V.A. et al. 2011 J. Nucl. Mater. 415 S365-8

[10] Allen S.L. et al. $201224^{\text {th }}$ IAEA FEC, PD 1-2

[11] Ryutov D.D. et al. 2008 Phys. of Plasmas 15092501

[12] Vijvers W.A.J. et al. Reduction of Peak Wall Power Loads in L-and H-mode Tokamak Plasmas in TCV with the Snowflake Divertor (poster) 2012, $24^{\text {th }}$ IAEA FEC, San Diego, California, USA

[13] Umansky M.V. et al. 2009 Nucl. Fusion 49075005

[14] Rognlien T.D. et al. 2013 J. Nucl. Mater. 438 S418

[15] Pericoli-Ridolfini V. et al. 2013 Fus. Eng. Des. 881677

[16] Ivanov A.A. et al. 200939 The SPIDER Code - Solution of Direct and Inverse Problems for Free Boundary Tokamak Plasma Equilibrium Keldysh Institute preprints

[17] Ryutov D.D. et al. 2010 Plasma Phys. Contr. Fusion 52105001

[18] Reimerdes H. et al. 2013 Plasma Phys. Contr. Fusion 55124027

[19] Reiter D. et al. 2005 Fus. Sci. Tec. 47 172-186

[20] Feng Y. et al. 2004 Contrib. Plasma Phys. 44, No. 1-3, 57-69

[21] Lunt T. el al. $200936^{\text {th }}$ EPS Conf. on Plasma Phys. Sofia, Bulgaria, June 29 - July 3, 2009 ECA Vol.33E, P-1.154

[22] Harting D. el al. 2011 J. Nucl. Mater. 415 S540-4

[23] Feng Y. el al. $201138^{\text {th }}$ EPS 2011 Conf. on Plasma Phys. Strasbourg, France, June 27 - July 1, $\mathrm{P} 1.071$

[24] Ryutov D.D. et al. 2012 Contrib. Plasma Phys. 52539

[25] Ryutov D.D. et al. 2012 Plasma Phys. Contr. Fusion 54124050

[26] Summers H.P., 1994 Atomic data and analysis structure users manual JET-IR 06 (Abingdon: JET Joint Undertaking). 


\begin{tabular}{l||r|r|r|r}
\hline$P_{\text {in }}[\mathrm{kW}]$ & 118.8 & 237.6 & 475.2 & 712.8 \\
\hline$P_{S P_{1}}[\%]$ & 28.7 & 27.3 & 29.8 & 31.1 \\
$P_{S P_{2}}[\%]$ & 0.5 & 0.4 & 0.7 & 0.8 \\
$P_{S P_{3}}[\%]$ & 0.8 & 0.2 & 1.0 & 1.3 \\
$P_{S P_{4}}[\%]$ & 33.1 & 39.4 & 44.3 & 45.8 \\
$\sum_{i} P_{S P_{i}}[\%]$ & 63.1 & 67.3 & 75.9 & 78.9 \\
Neutrals [\%] & 20.2 & 19.2 & 14.3 & 14.0 \\
Boundary [\%] & 16.0 & 13.1 & 9.3 & 7.0 \\
\hline
\end{tabular}

Table 1. Integrated power arriving strike points $\mathrm{SP}_{1}$ to $\mathrm{SP}_{4}$, normalized to the total input power $P_{i n}$, fraction of power transfered to the neutral particles (charge exchange and elastic collisions) and power fraction leaving the computational domain radially according to EMC3-Eirene simulations for four different input power values $P_{\text {in }}$. Transport coefficients $D_{\perp}=\chi_{\perp}=0.6 \mathrm{~m}^{2} / \mathrm{s}$ were assumed. 


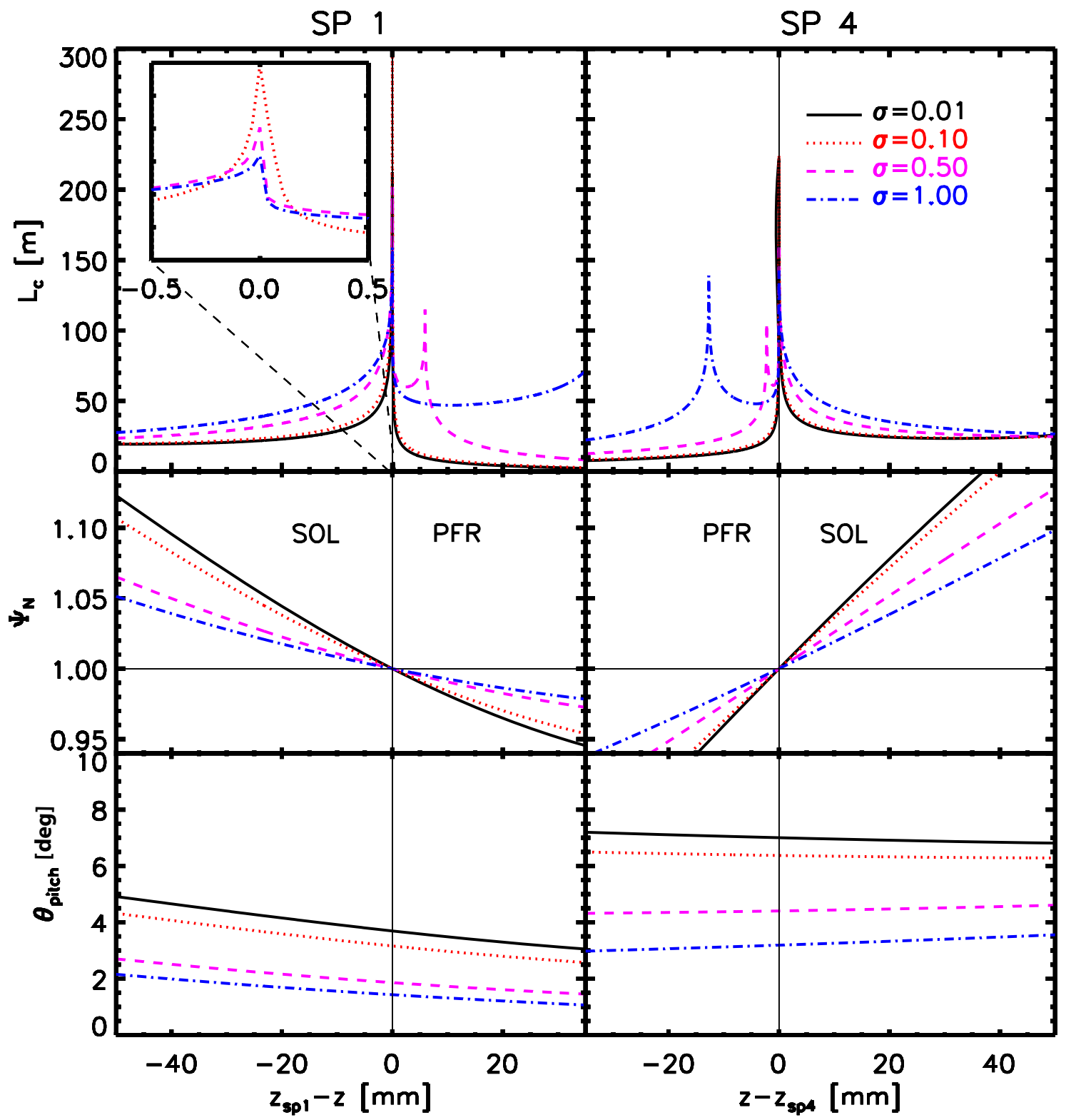

Figure 1. Connection length $L_{c}$, normalized poloidal flux $\Psi_{N}$ and $\theta_{\text {pitch }}$ at the divertor target plates as a function of the relative position of a starting point of a magnetic field line with respect to the primary strike point positions. 


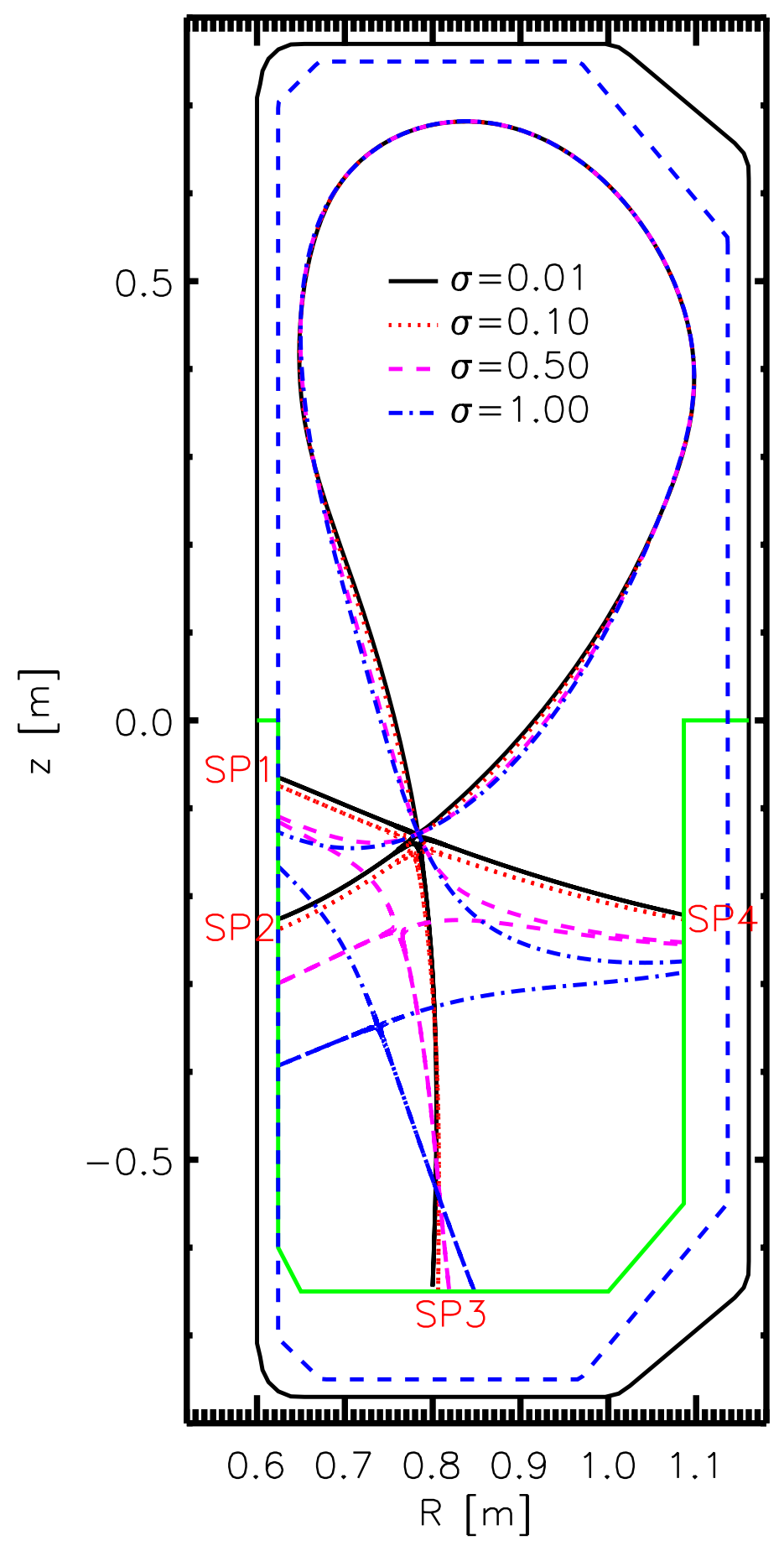

Figure 2. Separatrices of the four equilibria for different values of $\sigma$ ( $\sigma=$ $1.00,0.50,0.10$ and 0.01 ). The strike point positions $\mathrm{SP}_{1}$ to $\mathrm{SP}_{4}$ are labeled in counter clockwise direction as indicated here for the example of $\sigma=0.01$. 


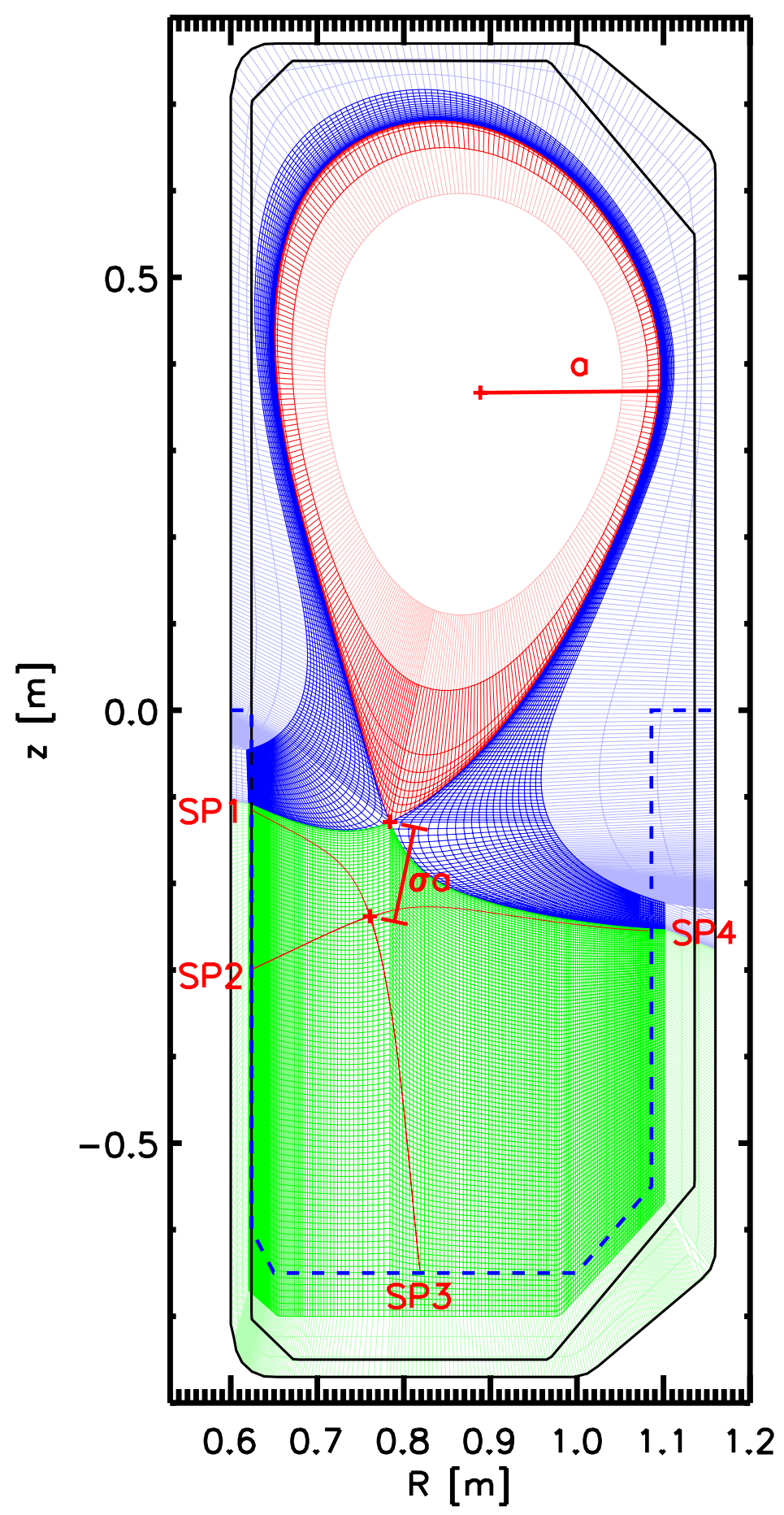

Figure 3. Poloidal cross section of the computational grid for EMC3-Eirene generated for a $\sigma=0.5$ configuration. In contrast to the core (red) and SOL (blue) regions the grid for the PFR (green) is not aligned with the flux surfaces. Instead of using the actual shape of the divertor tiles, the dashed blue curve was assumed for the divertor target due to technical reasons. 

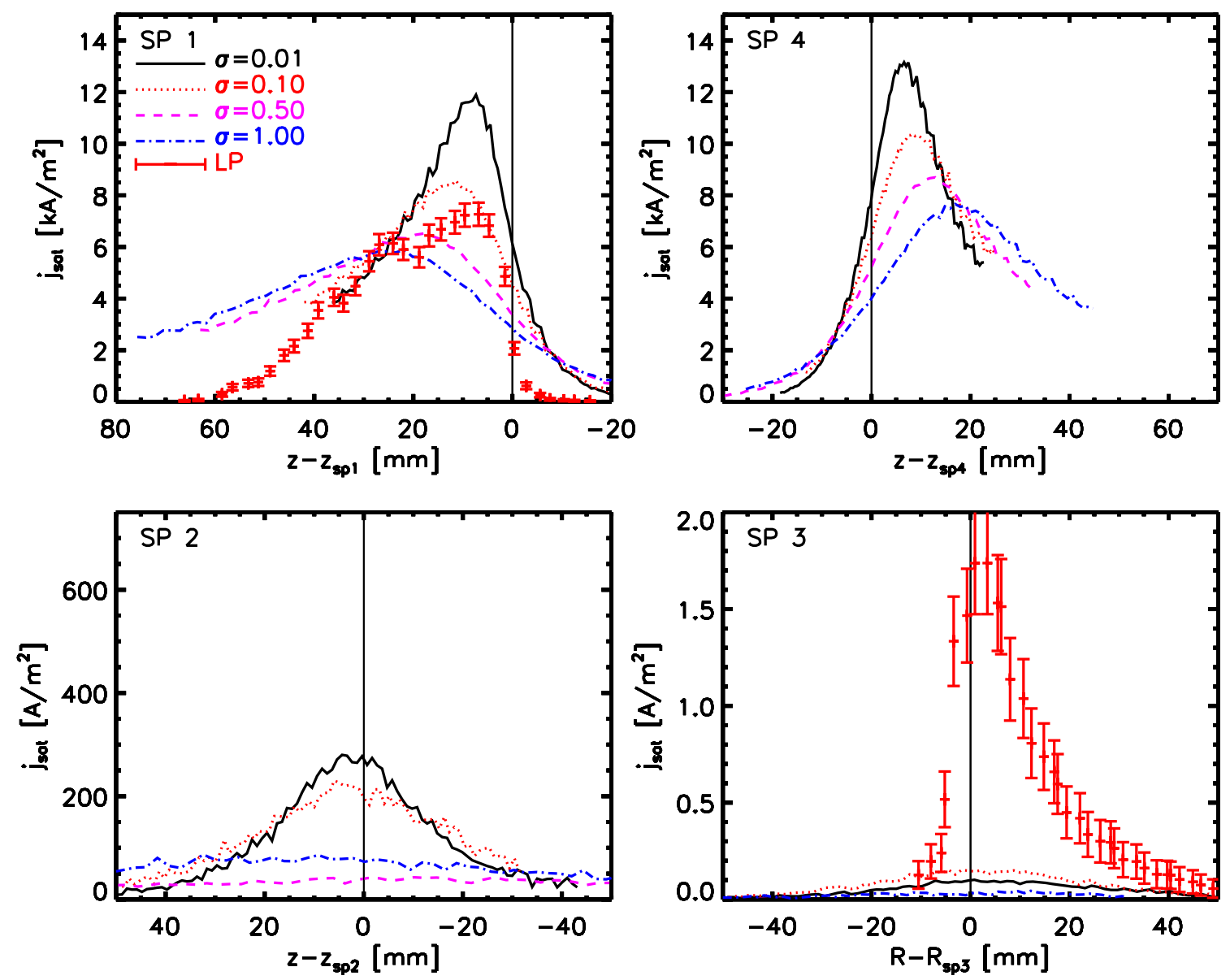

Figure 4. Particle deposition profiles for the 4 simulations with $\sigma=1.00,0.50,0.10$ and 0.01 at strike points labeled as $\mathrm{SP}_{1}-\mathrm{SP}_{4}$ (cf. Fig. 2). The lines correspond to the simulation, while the data points represent measurements by the divertor Langmuir probes for a $\sigma=0.1$ configuration. Note that the measured data for $\mathrm{SP}_{1}$ is shifted by $12 \mathrm{~mm}$, which is within the accuracy of the equilibrium reconstruction. 

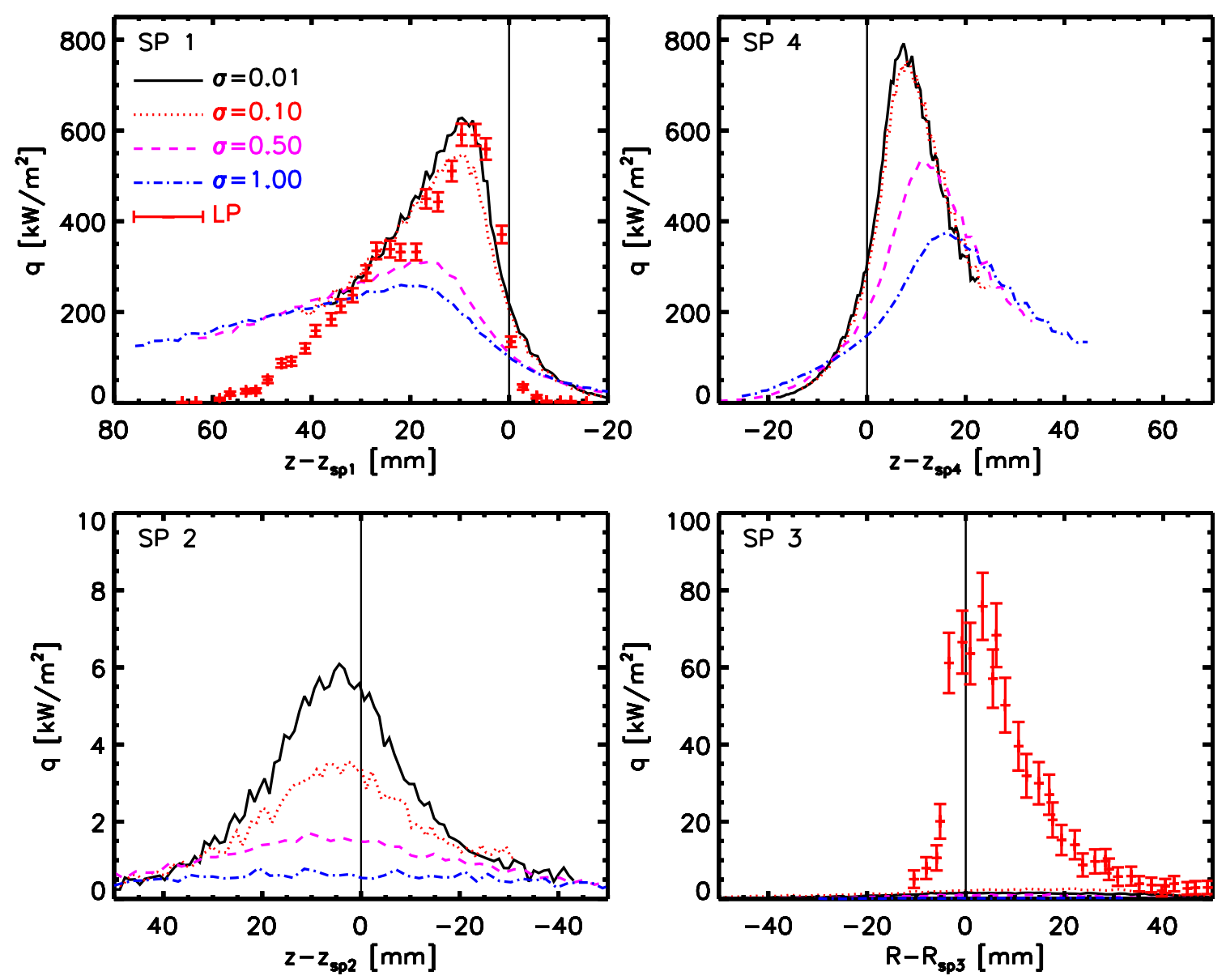

Figure 5. Same as Fig. 4 but for the power flux density $q$. 

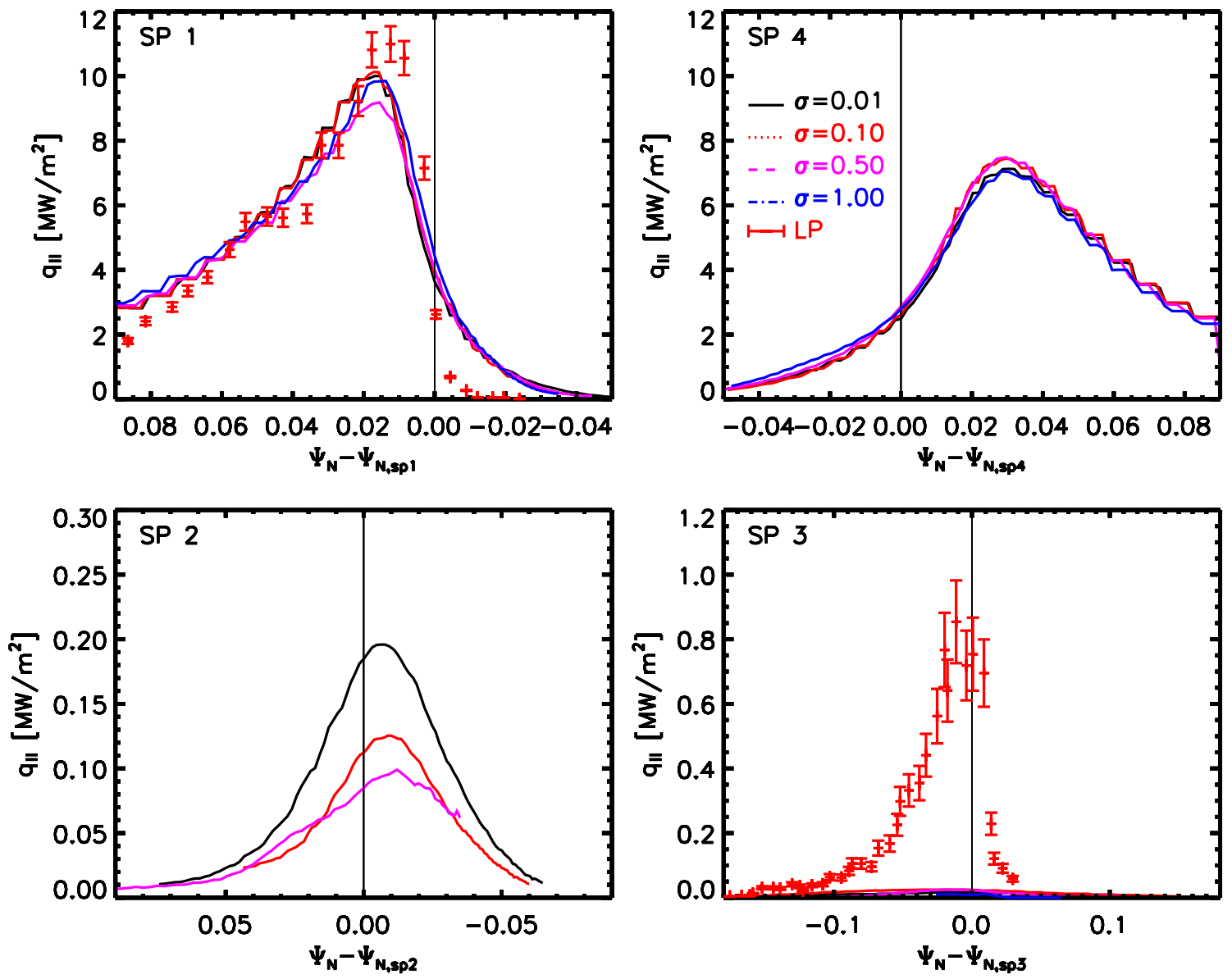

Figure 6. $q_{\|}$versus $\Psi-\Psi_{S P, i}$ for the same simulations as those of Figs. 4 and 5 . 


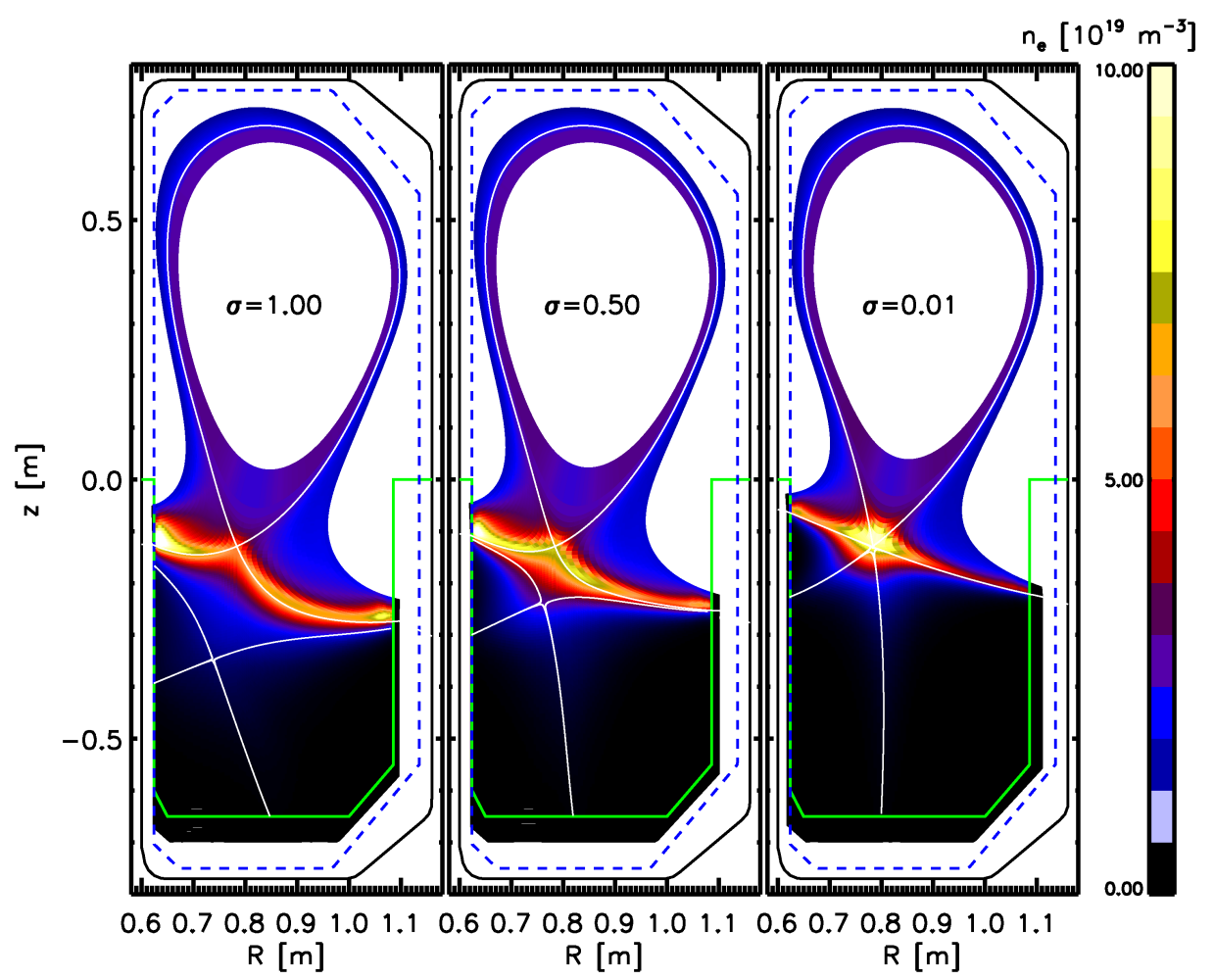

Figure 7. Formation of an x-point density blob during a $\sigma$-scan approaching the ideal snowflake configuration $(\sigma=0)$. The density on the separatrix point furthest away from the x-point is chosen to be $2 \cdot 10^{19} \mathrm{~m}^{-3}$ as a boundary condition kept fixed during the iteration process. The power through the inner simulation boundary is $P_{\text {in }}=P_{\text {heat }}=243 \mathrm{~kW}$. For the sake of brevity the plot for $\sigma=0.10$ is not shown here, since it looks almost identical to that for $\sigma=0.01$. 


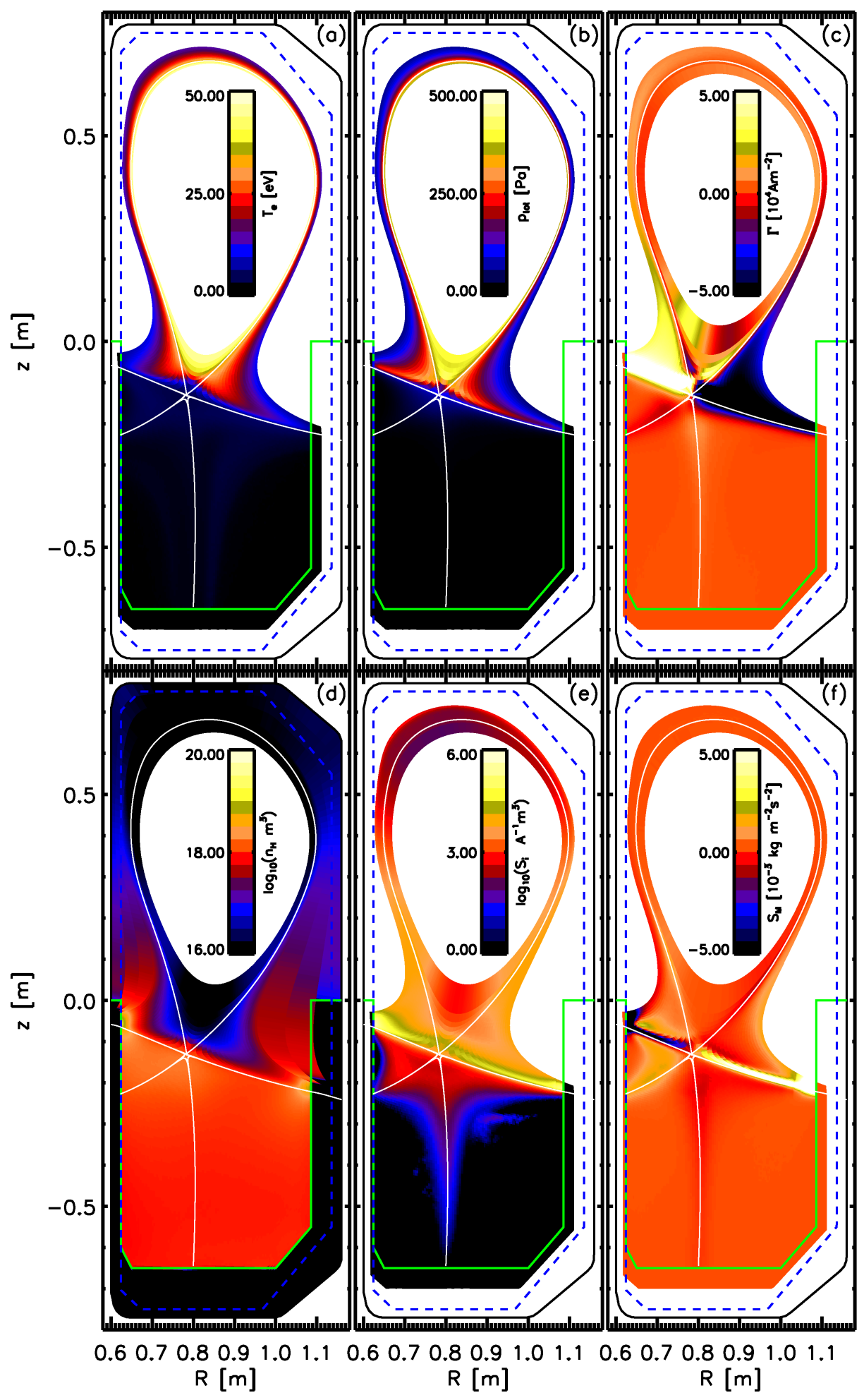

Figure 8. 2D poloidal profiles of (a) electron temperature $T_{e}$ (which is similar to that of the ions), (b) total pressure $p_{\text {tot }}=p_{\text {therm }}+P_{\text {kin }}=n_{e}\left(T_{e}+T_{i}\right)\left(1+M^{2}\right)$, (c) the particle flux density $\Gamma=n_{e} c_{s} M$, (d) atomic deuterium density $n_{H}$, (e) ionization strength $S_{i}$ and (f) the momentum loss $-S_{M}$ to the neutrals. 


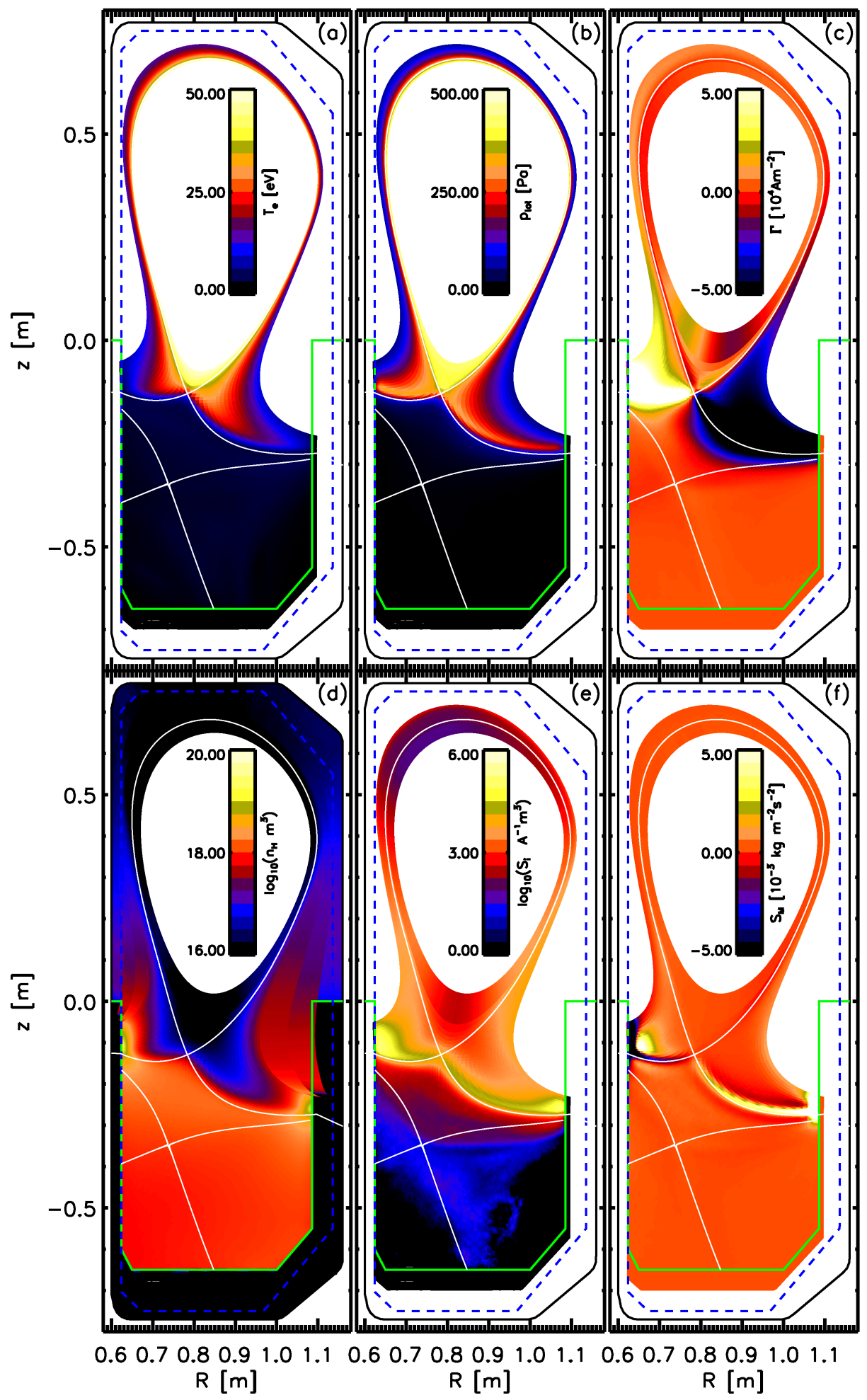

Figure 9. Same as Fig. 8 but for $\sigma=1$. 


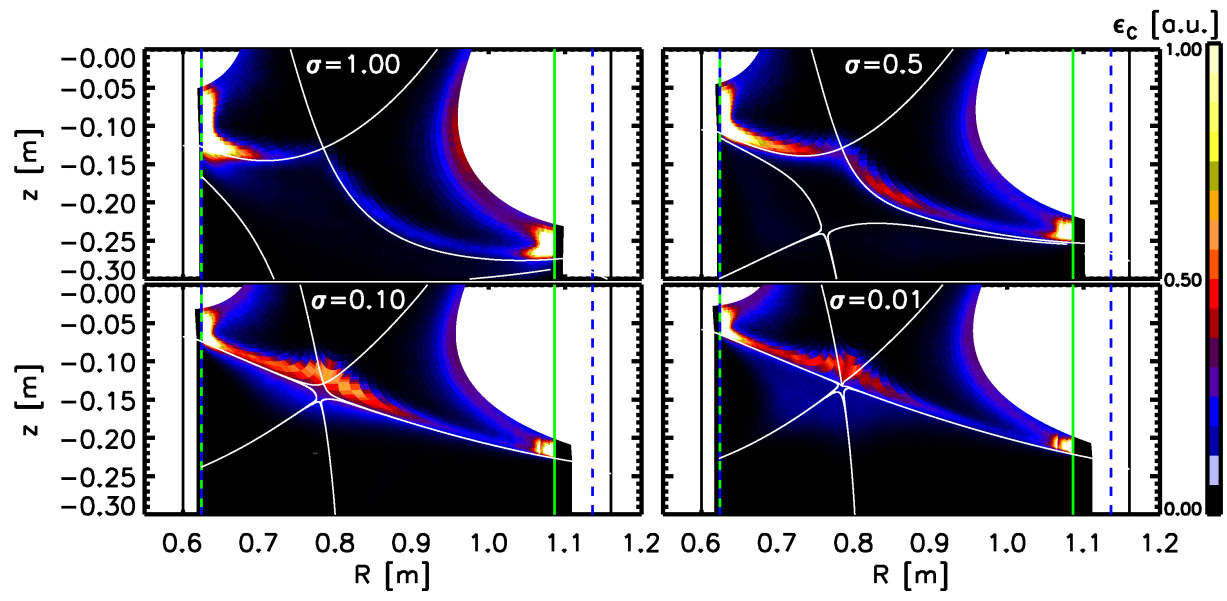

Figure 10. Carbon emissivity profile around the null point and divertor targets during a $\sigma$ scan assuming a constant sputtering yield $Y$.
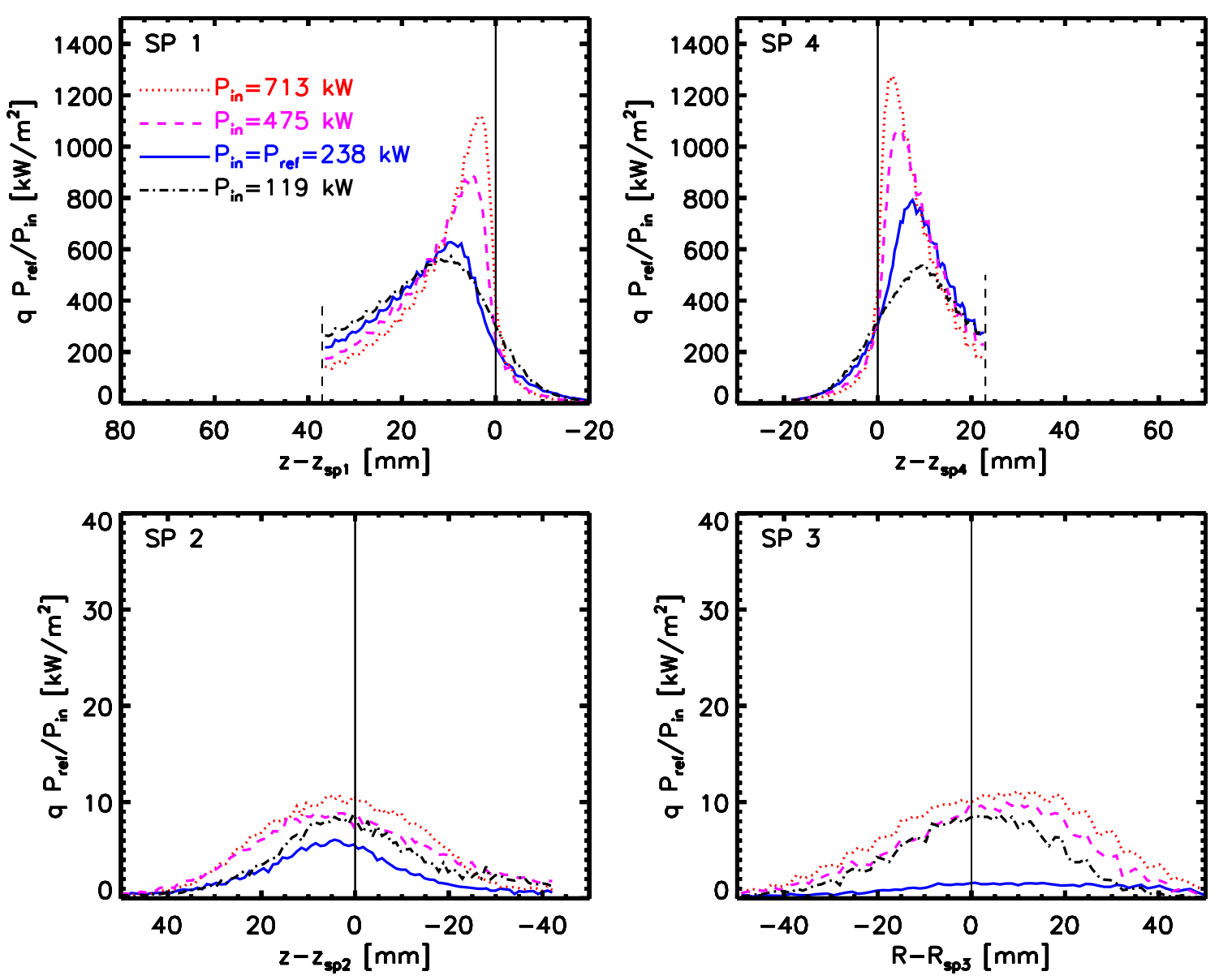

Figure 11. Power deposition profiles during an input power $P_{\text {in }}$ scan with $1 / 2,1$, 2 and 3 times the simulated reference discharge input power $P_{r e f}=238 \mathrm{~kW}$ for a $\sigma=0.01$ configuration. For the sake of comparability the profiles are scaled with the factor $P_{r e f} / P_{i n}$. A non-negligible part of the power $(7 \ldots 16 \%$, cf. Tab. 1) is leaving the computational boundary (dashed vertical line) which for technical reasons could not be extended radially further out so far. 


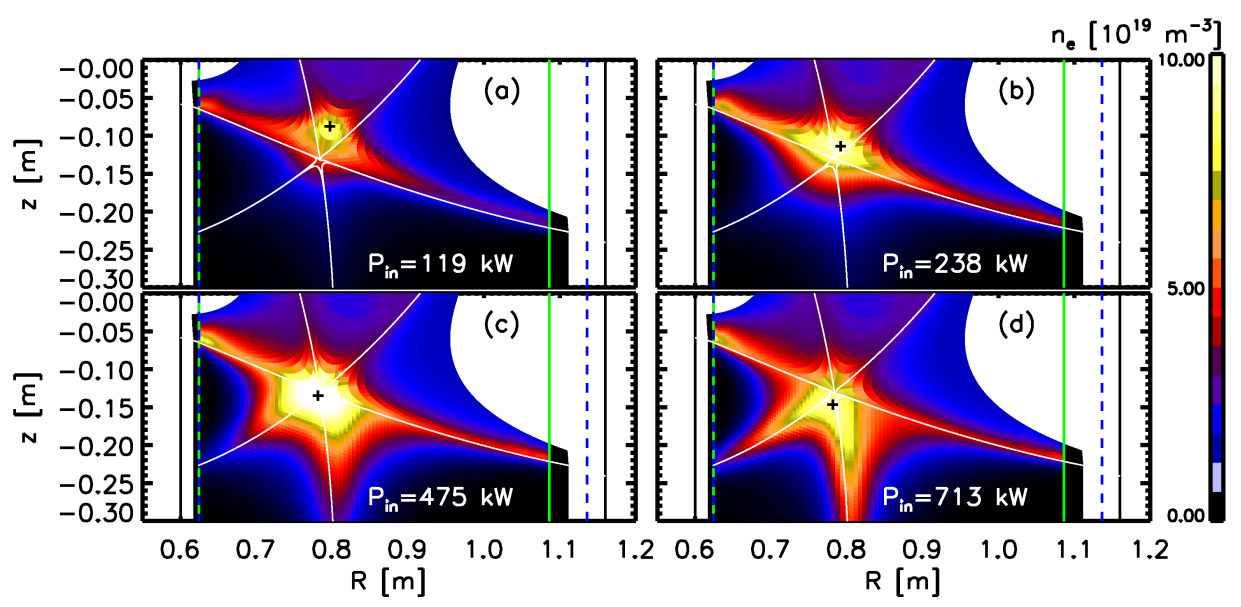

Figure 12. 2D plot of the density profile during a power scan with four different values of $P_{i n}$, (a) $122 \mathrm{~kW}$, (b) $243 \mathrm{~kW}$, (c) $486 \mathrm{~kW}$ and (d) $729 \mathrm{~kW}$. The position of the peak density indicated by the black cross is moving downwards as the input power increases. This peak value is (a) 3.6 (b) 4.5, (c) 5.5 and (d) 6.2 times higer than the minimum density on the separatrix. 